\title{
Method of Air Objects Status Flag Forming from Aggregate Radar Range Portraits with a Boardhand Sensing
}

Igor V. Sisigin,

Dmitriy A. Ravdin and Konstantin O. Kolesnikov*

Yaroslavl Higher Military Air Defence College

28 Moskovskiy, Yaroslavl, 150001, Russia

Received 12.12.2017, received in revised form 04.01.2018, accepted 27.02.2018

The article reveals the relevance of the assessment of air object in wideband sensing, suggestion the method of classification of the condition of object on the dynamics of their radar range portraits, described the method of forming aggregate radar range portraits from collapsing and maneuvering air targets with a boardband sensing, presented the results of mathematical simulation of collapsing and maneuvering aircrafts, proposed the structural scheme of the system of assessment of aerial objects on a correlation basis.

Keywords: modeling, detection of a condition of object, correlation coefficient, radar range portrait, wavelet transform.

Citation: Sisigin I.V., Ravdin D.A., Kolesnikov K.O. The method of forming aggregate radar range portraits from collapsing and maneuvering air targets with a boardband sensing, J. Sib. Fed. Univ. Eng. technol., 2018, 11(3), 325-335. DOI: 10.17516/1999494X-0045.

(C) Siberian Federal University. All rights reserved

* Corresponding author E-mail address: sisigin@mail.ru, ravdinda@yandex.ru 


\title{
Методика формирования признака \\ состояния воздушных объектов \\ по совокупности радиолокационных \\ дальностных портретов \\ при широкополосном зондировании
}

\author{
И.В. Сисигин, Д.А. Равдин, К.О. Колесников \\ Ярославское высшее военное училище \\ противовоздушной оборонь \\ Россия, 150001, Ярославль, пр. Московский, 28
}

В статье раскрывается актуальность оценки состояния воздушных объектов при широкополосном зондировании, предложен вариант классификации состояния воздушных объектов по динамике их радиолокачионных дальностных портретов, описана методика формирования совокупности радиолокационных дальностных портретов маневрирующих и разрушаюшчхся воздушных объектов при широкополосном зондировании, представлены результаты математического моделирования маневрирующих и разрушающцхся воздушных объектов.

Ключевые слова: моделирование, распознавание состояния воздушного объекта, коэффициент корреляции, радиолокационный дальностный портрет, вейвлет-преобразование.

В $[1,2]$ говорится, что наряду с традиционными (обнаружение и разрешение целей, измерение координат и параметров их движения) в число основных задач радиолокационного наблюдения выдвинулось распознавание классов, типов и функционального состояния воздушных объектов (BO).

В [3-5] достаточно широко представлены методы и алгоритмы распознавания классов и типов ВО. В [6] предложен метод распознавания маневра ВО, но не рассматривается процесс их разрушения. В $[7,8]$ автор предлагает способ определения момента фрагментации объекта, но рассматривает только процесс кинетического взаимодействия космических аппаратов с астероидами и другим объектами. Однако оценка функционального состояния аэродинамических ВО радиолокационными методами остается нерешенной, поэтому актуальность рассматриваемой темы достаточно очевидна.

Под функциональным состоянием воздушного объекта понимается его положение в пространстве по отношению к радиолокационной станции (РЛС) (прямолинейное движение или совершение какого-либо маневра) и геометрическое расположение его составных частей по отношению друг к другу (разрушение или разделение), которое может быть определено по отраженному сигналу.

Как известно, отраженный от цели сигнал при высоком разрешении имеет достаточно сложную временную структуру, которая определяется геометрическими и электродинамическими особенностями. Такой сигнал после согласованной обработки и амплитудного детектирования дает радиолокационный дальностный портрет (РЛДП). Реализация РЛДП зависит не только от геометрической формы воздушного объекта (ВО), но и от ориентации его элементов 
относительно линии визирования РЛС. Поэтому нами выдвинута гипотеза о том, что РЛДП ВО как при маневре, так и при разрушении претерпевает существенные изменения, однако при маневре они могут носить регулярный характер, а при разрушении - хаотичный. Анализ динамики РЛДП позволит классифицировать ВО по состоянию: прямолинейное движение/маневр/ разрушение (рис. 1).

Для проверки выдвинутой гипотезы были рассмотрены три возможных состояния ВО: прямолинейное движение, маневр и разрушение.

Для анализа был выбран метод математического моделирования, основанный на преобразовании 3D-моделей ВО в дифференциальную эффективную поверхность рассеяния (ЭПР), свертки ее с зондирующим сигналом и последующей согласованной обработки с вычислением модуля выходного сигнала (рис. 2), который подробно описан в [9].

Динамика РЛДП оценивалась по признаку в виде коэффициента корреляции (КК) между текущим и предыдущим РЛДП в соответствии с выражением

$$
r_{x y}=\frac{\sum\left(x_{k}-\bar{x}\right)\left(y_{k}-\bar{y}\right)}{\sqrt{\sum\left(x_{k}-\bar{x}\right)^{2} \sum\left(y_{k}-\bar{y}\right)^{2}}}
$$

где $x_{k}$ - РЛДП ВО в момент времени $t_{1} ; \bar{x}$ - среднее значение $x$ по выборке $X ; y_{k}$ - РЛДП ВО в момент времени $t_{2} ; \bar{y}$ - среднее значение $y$ по выборке $Y$.

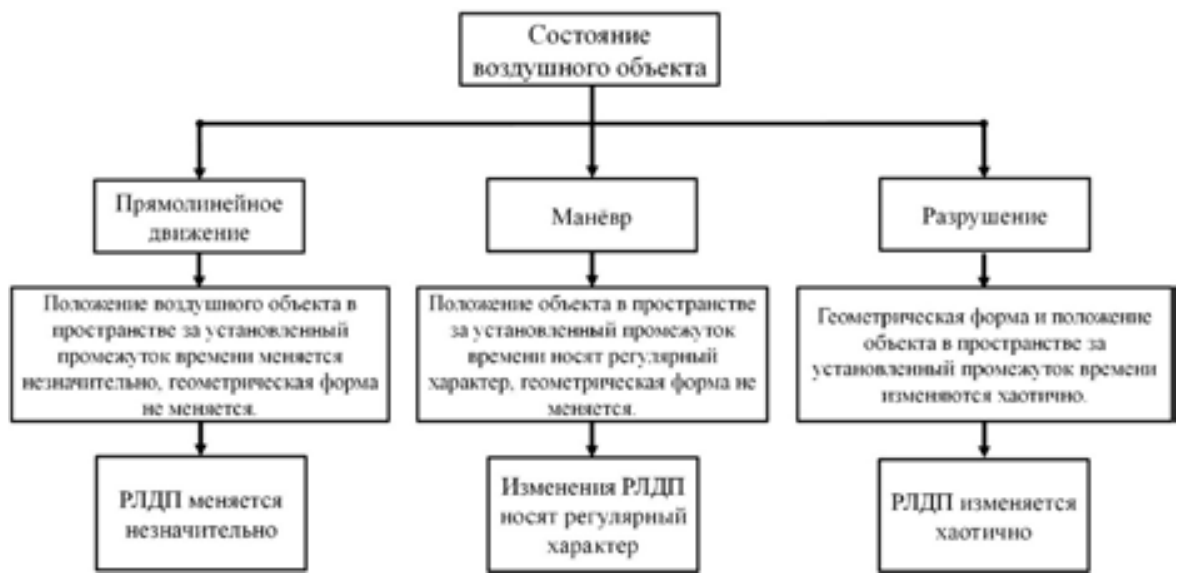

Рис. 1. Классификация состояния воздушных объектов и описание признаков на основе РЛДП

Fig. 1. Classification of the condition of the objects and a description of the characteristics based on radar longrange portraits (RLRP)

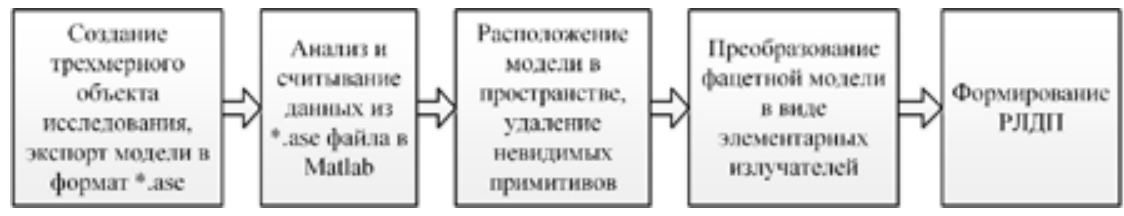

Рис. 2. Этапы математического моделирования РЛДП

Fig. 2. Stages of RLRP mathematical modeling 
Для проведения эксперимента были разработаны модели процессов разрушения и маневра типового воздушного объекта.

Процесс разрушения ВО в результате кинетического воздействия либо технической неисправности является случайным, поэтому для его анализа были использованы видеоматериалы реальных событий с ВО. В качестве прототипа варианта разрушения был взят видеофайл, отдельные кадры которого представлены на рис. 3.

Время анализа ограничено одной секундой, так как за этот промежуток происходят существенные изменения геометрической формы ВО. На основе имеющихся видеоматериалов в программе 3Dmax разработаны покадровые модели BO, соответствующие по характеру изменения геометрической формы объекту из видеофайла (рис. 4).

Формирование покадровой последовательности из 25 РЛДП проводилось описанным выше методом математического моделирования [9].

На рис. 5 и 6 представлены совокупности РЛДП разрушающегося ВО №1 при сигналах с шириной спектра (ШС) 30 и 300 МГц соответственно.

Для представленных на рис. 5, 6 совокупностей РЛДП по формуле (1) рассчитаны значения КК. Минимальное значение КК при сигнале с ШС 30 МГц составило 0,95, при сигнале с

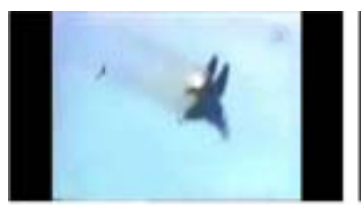

0 кадр $(0,0 \mathrm{c})$

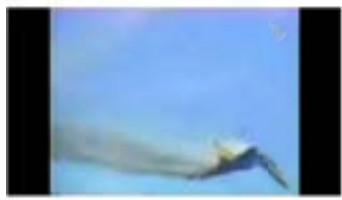

15 калр $(0,6$ c)

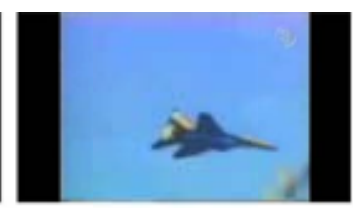

5 кадр $(0,2$ c)

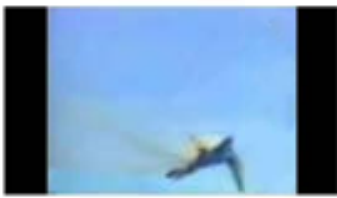

20 калц $(0,8 \mathrm{c})$

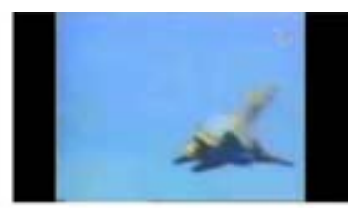

10 кадр $(0,4$ c)

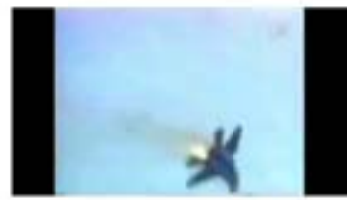

24 калр $(1,0 \mathrm{c})$

Рис. 3. Отдельные кадры процесса разрушения ВО

Fig. 3. Individual frames of the process of destruction of aircraft object (AO)

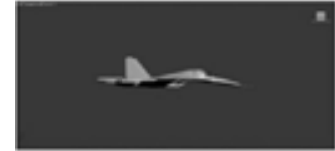

1 кадр

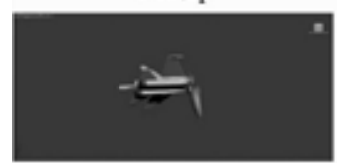

15 калр

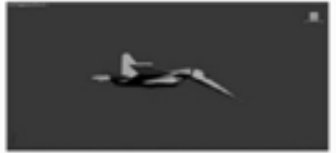

5 калр

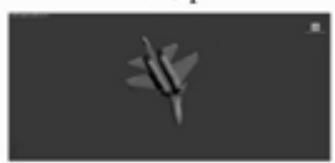

20 калр

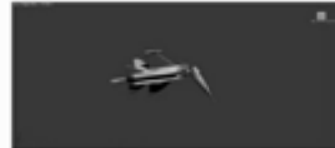

10 калр

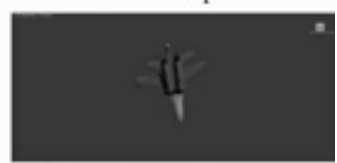

24 катр

Рис. 4. 3D-модели разрушения воздушного объекта №1

Fig. 4. 3D-models of destruction of air object №1 


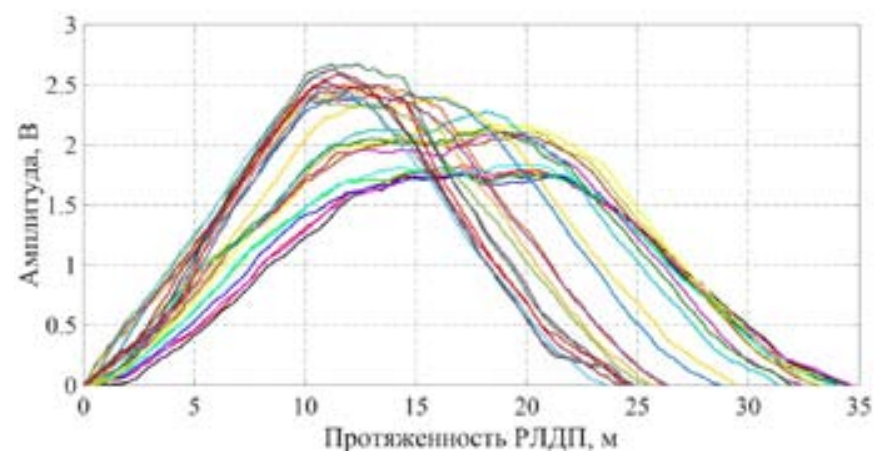

Рис. 5. Совокупность РЛДП разрушающегося ВО №1 при сигнале с ШС 30 МГц

Fig. 5. Set of RLRP of the collapsing air object №1 at the signal with a spectrum width of $30 \mathrm{MHz}$

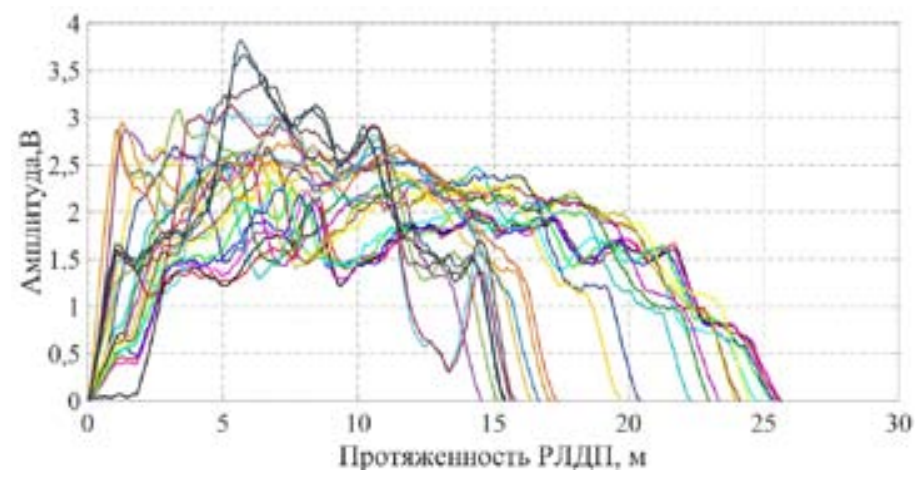

Рис. 6. Совокупность РЛДП разрушающегося ВО №1 при сигнале с ШС 300 МГц

Fig. 6. Set of RLRP of the collapsing air object №1 at the signal with a spectrum width of $300 \mathrm{MHz}$

ШС 300 МГц - 0,72. Для получения достаточной статистики было реализовано несколько вариантов разрушения четырех типов ВО с различных ракурсов. Во всех экспериментах рузультаты имели достаточную сходимость: среднее значение КК при сигнале с ШС 30 МГц составило не менее 0,9 , а для сигнала с ШС300 МГц - не менее 0,7 .

Маневр воздушного объекта - это процесс, близкий к детерминированному. Поэтому его можно представить в виде движения по окружности радиусом $R$ (рис. 7).

В случае пилотируемого полета предельное значение длительной перегрузки не может превышать значения $10 \mathrm{~g}$, что соответствует пределу физиологических возможностей человека.

При этом связь между параметрами движения и радиусом кривизны траектории $R$ определяется выражением

$$
a_{\amalg}=\frac{V_{\amalg}^{2}}{R}=\omega_{\amalg}^{2} R,
$$

где $V_{\text {ц }}$ - тангенциальная составляющая скорости объекта в точке начала маневра; $\omega_{\text {ц }}-$ угловая скорость движения объекта по окружности; $R$ - радиус кривизны траектории движения объекта. 


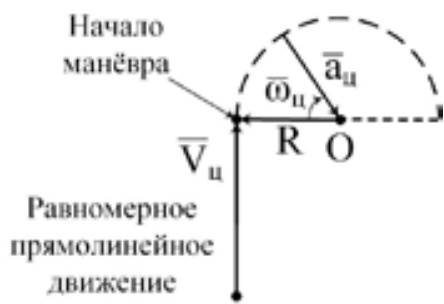

Рис. 7. Модель выполнения процесса маневра ВО

Fig. 7. The execution model of the AO maneuver process

Таблица 1

Table 1

\begin{tabular}{|c|c|c|c|c|c|c|c|c|c|c|}
\hline$V_{\text {}}, \mathrm{M} / \mathrm{c}$ & 100 & 200 & 300 & 400 & 500 & 600 & 700 & 800 & 900 & 1000 \\
\hline $\mathrm{R}, \mathrm{M}$ & 102 & 408 & 918 & 1632 & 2551 & 3673 & 5000 & 6530 & 8265 & 10204 \\
\hline
\end{tabular}

С учетом перегрузки $n$ - отношения абсолютной величины линейного ускорения цели $a_{\Perp}$, вызванного негравитационными силами, к ускорению свободного падения $g$, имеем:

$$
\begin{aligned}
& n=\frac{a_{\text {щ }}}{g},
\end{aligned}
$$

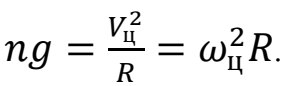

Таким образом, угловая скорость вращения относительно РЛС, которая приводит к изменению РЛДП, зависит от тангенциальной составляющей скорости цели и допустимых значений перегрузки. Рассчитанные радиусы кривизны траектории маневра для скоростей ВО от $100 \mathrm{~m} / \mathrm{c}$ до 1000 м/с представлены в таблице 1.

Дальнейшие результаты представлены для модели маневра при скорости движения ВО в 300 м/с. Исходя из этого скорость $\omega$ будет равна 0,33 рад/с, что соответствует повороту ЛА относительно РЛС со скоростью $19 \%$.

На рис. 8 и 9 показаны совокупности РЛДП маневрирующего ВО №1 при углах курса $\beta=0-19^{\circ}$ с шагом $1^{\circ}$ и ширине спектра зондирующего сигнала 30 и 300 МГц соответственно.

Для представленных на рис. 8 и 9 совокупностей РЛДП по формуле (2) рассчитаны значения коэффициентов корреляции между текущим и предыдущим реализациями РЛДП. Все значения коэффициентов корреляции лежат в пределах значений 0,9-0,99.

На рис. 10 показаны графики изменения коэффициентов корреляции ВО №1 для сигналов с ШС 30 и 300 МГц для маневра и разрушения.

На рис. 10 видно, что КК РЛДП не опускается ниже уровня 0,7 при сигнале с ШС 300 МГц, а при сигнале с ШС 30 МГц - не ниже 0,95 .

Отношение коэффициентов корреляции при маневре и разрушении составили 1,04 и 1,36 для 30 и 300 МГц соответственно, что свидетельствует о малой контрастности признака. 


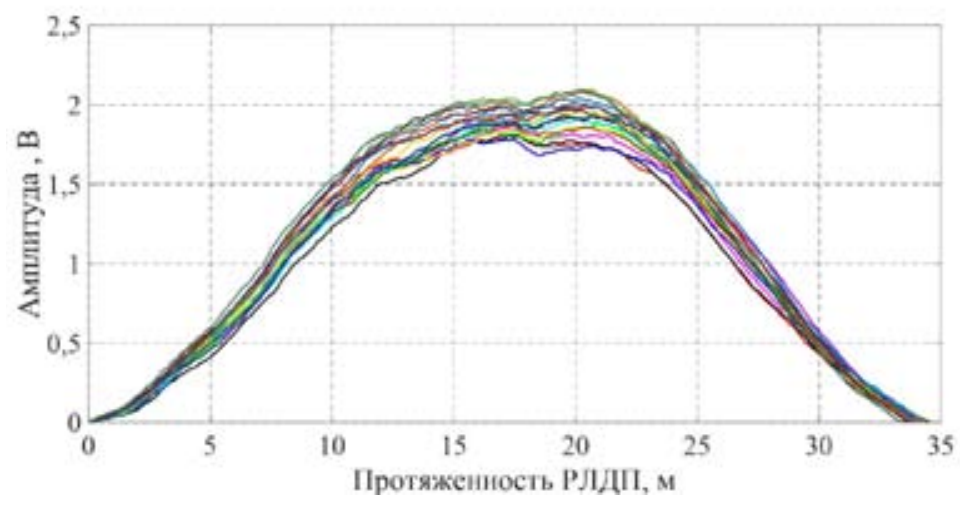

Рис. 8. Совокупность РЛДП маневрирующего ВО №1 при углах курса $\beta=0^{\circ}-19^{\circ}$ для сигнала с ШС ширине спектра 30 МГц

Fig. 8. Set of RLDP for maneuvering AO №1 at angles of $\beta=0^{\circ}-19^{\circ}$ for signal spectrum width spectrum width of $30 \mathrm{MHz}$

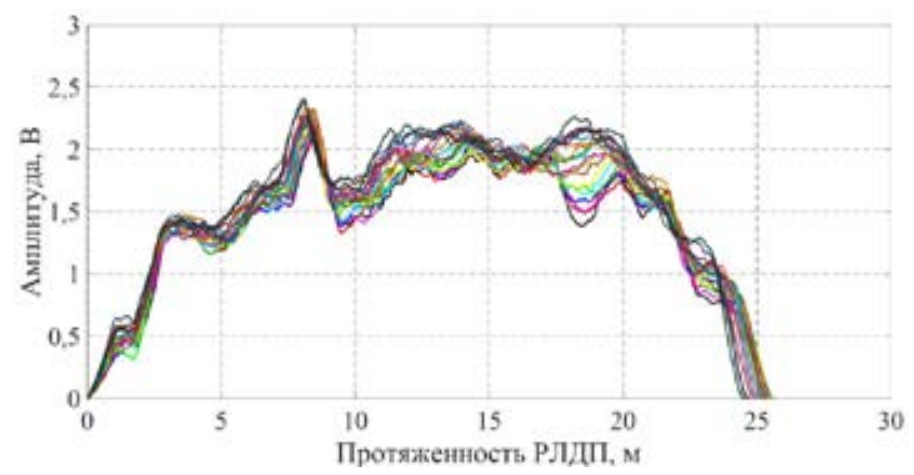

Рис. 9. Совокупность РЛДП маневрирующего ВО №1 при углах курса $\beta=0-19^{\circ}$ для сигнала с ШС ширине спектра $300 \mathrm{MГц}$

Fig. 9. Set of RLDP for maneuvering $A O$ №1 at angles of $\beta=0^{\circ}-19^{\circ}$ for signal spectrum width spectrum width of $300 \mathrm{MHz}$

Таким образом, гипотеза о существенном изменении РЛДП при маневре и разрушении подтвердилась, однако значения выбранного признака оказались невелики, поэтому для повышения контрастности выбранного признака (КК) целесообразно провести дополнительную обработку.

Для решения этой задачи была использована двухэтапная процедура вейвлетпреобразования, предложенная в [5] для формирования контрастных признаков распознавания радиолокационных целей.

На первом этапе для каждого РЛДП вычисляются вейвлет-коэффициенты $W_{z}(a, b)$ в соответствии с выражением

$$
W_{z}(a, b)=\frac{1}{\sqrt{a}} \int_{-\infty}^{\infty} Z(t) \Psi\left(\frac{t-b}{a}\right) d t
$$




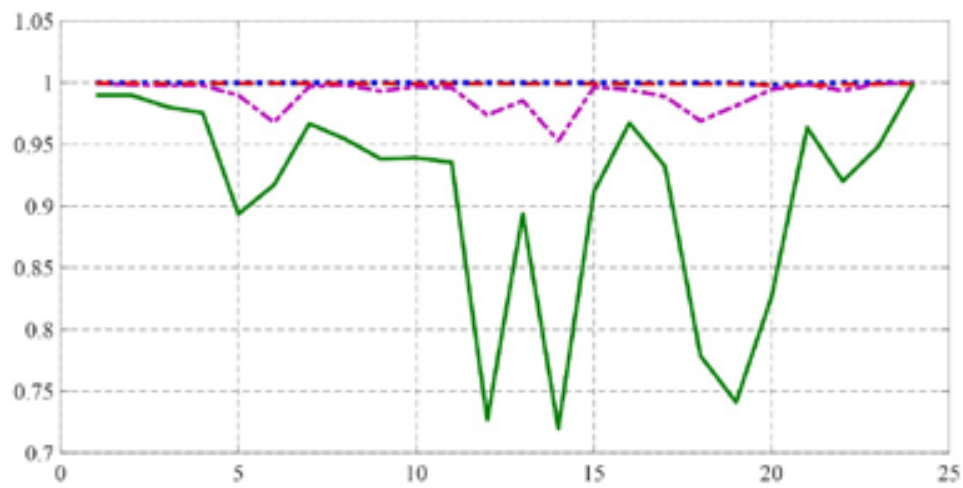

РЛДП Су-34

маневр $30 \mathrm{MГи}$

ргли Сy-34

мatresp $300 \mathrm{M \Gamma n}$

РлдП Су-34

разрушение

$30 \mathrm{M \Gamma u}$

Рлдп Су-34

разрушение

$300 \mathrm{MГц}$

Рис. 10. График изменения КК совокупности РЛДП ВО №1 при сигнале с ШС 30 и 300 МГц для маневра и разрушения

Fig. 10. Graph of changes in the correlation coefficients of the set of RLRP of the air object №1 at a signal with a spectrum width of $30 \mathrm{MHz}$ and $300 \mathrm{MHz}$ for maneuvering and destruction

где $Z(t)$ - входной РЛДП; $a$ - параметр масштаба; $b$ - параметр временного переноса; $\Psi\left(\frac{t-b}{a}\right)$ базисная функция Хаара.

На втором этапе из $W_{z}(a, b)$ формируются $\operatorname{LLE}(a, b)$ - линии локальных экстремумов (ЛЛЭ) в соответствии с выражением

$$
L L E(a, b)=\left\{\begin{array}{l}
1,\left|\frac{d W_{z}(a, b)}{d b}\right| \leq \Delta W \\
0,\left|\frac{d W_{z}(a, b)}{d b}\right|>\Delta W
\end{array},\right.
$$

где $\Delta W$ - заданное пороговое значение скорости изменения функции $W_{z}(a, b)$.

На рис. 11 представлены совокупности ЛЛЭ ВО №1 при сигнале с ШС 30 МГц для маневра и разрушения.

Характерной особенностью ЛЛЭ является их компактность и малая дисперсия при регулярном изменении РЛДП (рис. 11a). При разрушении цели ЛЛЭ изменяются значительно больше (рис. 11б).

Для оценки динамики коэффициента корреляции ЛЛЭ при маневре и разрушении был проведен статистический эксперимент, результаты которого изображены на рис. 12 и 13.

Из графиков на рис. 12, 13 видно, что среднее значение $\mathrm{KK}_{L L E}$ при маневре более чем в два раза превышает значение $\mathrm{KK}_{L L E}$ при разрушении для сигналов с ШС 30 и 300 МГц, а среднеквадратичное отклонение $\mathrm{KK}_{L L E}$ при маневре в 1,5 раза меньше, чем при разрушении для сигнала с ШС 30 МГц и в 3 раза меньше для сигнала с ШС 300 МГц $(0,13$ / 0,19; 0,06 / 0,2 соответственно).

Таким образом, предложенная на рис. 14 методика формирования признака маневра/разрушения, основанная на вычислении линий локальных экстремумов от вейвлет-коэффициентов РЛДП, обеспечила его высокую контрастность, что может повысить качество распознавания в задаче оценки функционального состояния объектов. 


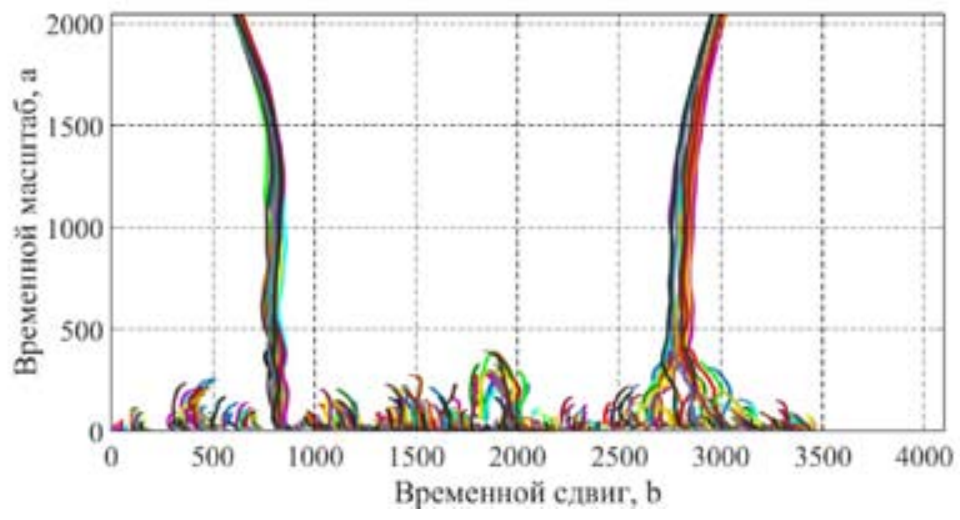

a)

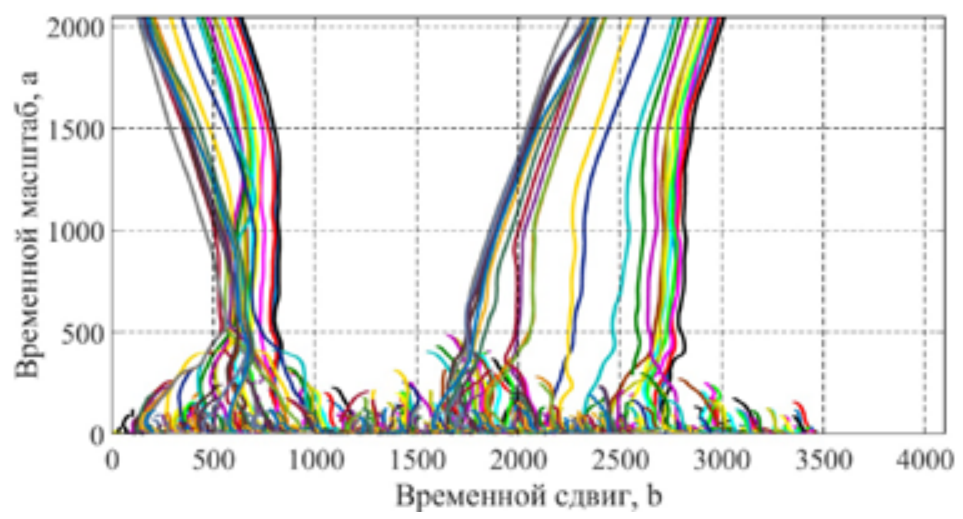

б)

Рис. 11. Совокупность ЛЛЭ ВО №1: а - при маневре $\left(\beta=0-19^{\circ}\right) ;$ б - при разрушении

Fig. 11. Set of lines the local extrema (LLE): a - when maneuvering $\left(\beta=0-19^{\circ}\right)$; b) with the destruction

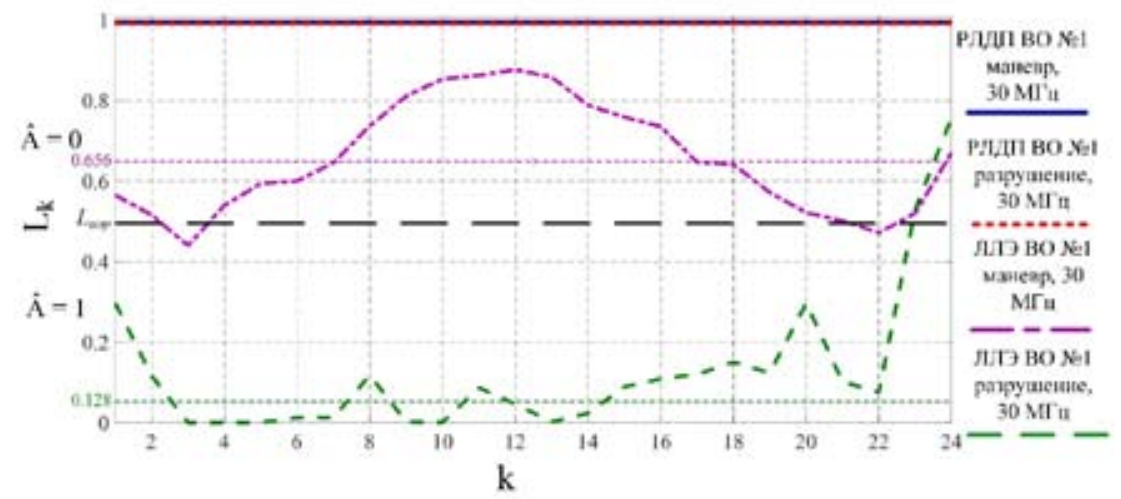

Рис. 12. График изменения КК совокупности РЛДП и ЛЛЭ для маневра и разрушения при сигнале с ШС $30 \mathrm{MГц}$

Fig. 12. Graph of changes in correlation coefficients of the set of RLRP and LLE for maneuvering and destruction at a signal with a spectrum width of $30 \mathrm{MHz}$ 


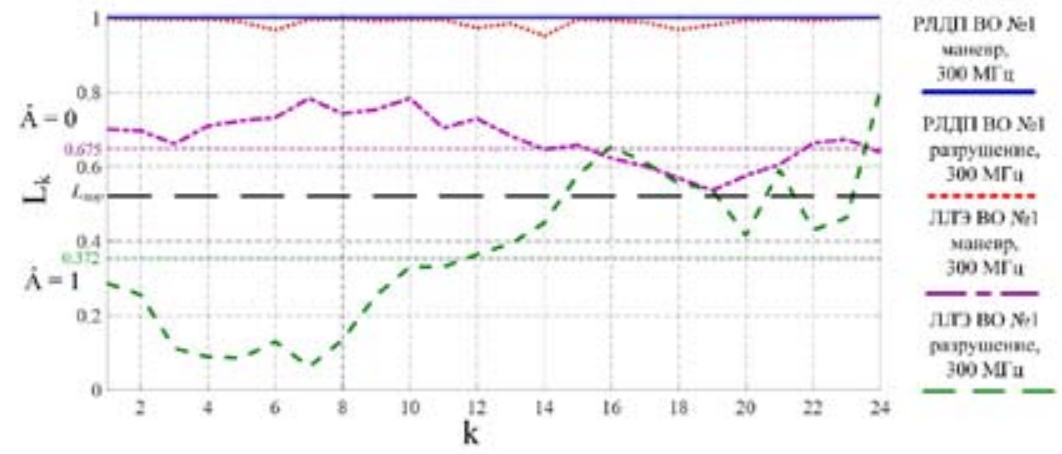

Рис. 13. График изменения КК совокупности РЛДП и ЛЛЭ ВО №1 для маневра и разрушения при сигнале с ШС 300 МГц

Fig. 13. Graph of the correlation coefficients of the set of RLRP and LLE to maneuver and destruction with a spectrum width of $30 \mathrm{MHz}$

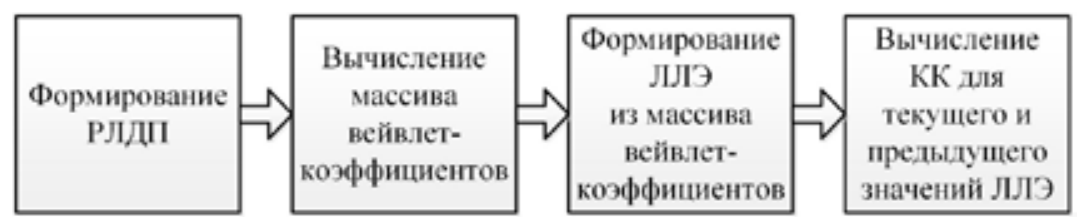

Рис. 14. Структурная схема методики формирования признака маневра/разрушения

Fig. 14. Structural scheme of the formation method of maneuver/destruction sign

\section{Список литературы}

[1] Слюсарь Н.М. Эффект вторичной модуляции радиолокационных сигналов: физические основы и практическое применение, Вестник Военной академии Республики Беларусь, 2003, 1, 61-77. [Slyusar N.M. Effect of secondary modulation of radar signals: physical basis and practical application, Bulletin of the military Academy of the Republic of Belarus, 2003. 1. 61-77. (in Belarus)]

[2] Слюсарь Н.М. Радиолокаиионное распознавание, контроль состояний и действий иелей в радиотехнических системах вооружения на основе вторичной модуляции отраженных сигналов. Минск: ВА РБ, 2003. 144. [Slyusar N.M. Radar detection, control of States and actions goals, in electronic weapons systems on the basis of secondary modulation of the reflected signals. Minsk: VA RB, 2003. 144. (in Belarus)]

[3] Богданов А.В., Бондарев В.Н., Васильев О.В., Гарин Е.Н. и др. Синтез оптимальных алгоритмов распознавания групповых воздушных целей в радиолокационных системах воздушного базирования. Журнал Сибирского федерального ун-та. Техника и технологии 2017, 10(2). 155-168. [Bogdanov A.V., Bondarev V.N., Vasiliev O.V., Garin E.N. and other. Synthesis of optimal algorithms for recognition of group air targets in air-based radar systems. Journal Siberian Federal University, Engineering and Technology, 2017, 10(2). 155-168. (in Russia)]

[4] Ищук И.Н., Степанов Е.А., Бебенин А.А. и др. Способ классификации объектов оптикоэлектронными системами разведки на основе обработки многоспектрального кубоида изображений. Журнал Сибирского федерального ун-та. Техника и технологии, 2017, 10(2), 183-190. [Ischuk I.N., Stepanov E.A., Bebenin A.A. etc. Method of classification of objects by electro-optical 
intelligence systems based on the processing of multispectral cuboid images. Journal Siberian Federal University, Engineering and Technology, 2017, 10(2), 183-190. (in Russia)]

[5] Колесников К.О., Сисигин И.В., Равдин Д.А. и др. Методика формирования признаков распознавания на основе вейвлет-преобразования радиолокационных дальностных портретов. Журнал Сибирского федерального ун-та. Техника и технологии, 2017, 10(2), 277-285. [Kolesnikov K.O., Sisigin I.V., Ravdin D.A. and others. Methods of forming the signs of recognition based on wavelet transform radar dolnestic portraits. Journal Siberian Federal University, Engineering and Technology, 2017, 10(2), 277-285. (in Russia)]

[6] Ковтунов А.Л. Лещенко С.П., Батуринський М.П., Польшина Л.В. Метод обнаружения маневра радиолокационной цели в обзорных РЛС с использованием сверхширокополосных сигналов. Збірник наукових прачь Харківського університету Повітряных Сил, 2013, 3(36). 5559. [Kovtunov A.L. Leshchenko S.P., Baturinsky M.P., Pollina L.V. Method of maneuver detection in radar target surveillance radar using ultra-wideband signals. Collection of scientific papers of Kharkiv University Air Forces, 2013, 3(36). 55-59. (in Ukraine)]

[7] Антропова С.М. Моделирование одного из подходов к решению задачи оценки факта поражения. Сборник докладов НТК «ВВТ ВКО» в МГТУ имени Н.Э. Баумана, 2014. [Antropova S.M. Modeling of one of the approaches to the problem of assessment of the fact of defeat. Collection of reports of STC "BBT EKR” in MSTU named After N. Uh... Bauman, 2014 (in Russia)]

[8] Антропова С.М. Исследования фрагментации объекта для решения задач контроля факта поражения. Сборник докладов 5-й НTК «Актуальные вопросы развития систем и средств ВКО», 2015. [Antropova S.M. Study of the fragmentation of the object for the decision of tasks of control of the fact of defeat. Collection of papers 5-th NTK "Topical issues of development of systems and tools, ASD”, 2015. (in Russia)]

[9] Колесников К.О. Сисигин И.В. Равдин Д.А., Сушин Ю.В. Методика моделирования отраженных сигналов от сложных объектов на основе приближения Гюйгенса-Френеля. Вестник воздушно-космической обороны, 2014. [Kolesnikov K.O., Sisigin I.V., Ravdin D.A., Sushin Yu.V. Modeling reflected signals from complex objects on the basis of approximation of the Huygens-Fresnel. Journal of Aerospace Defense, 2014 (in Russia)] 